\title{
The Canonical Order and Optimization Problems
}

\author{
Michail M. Kovalev AND DMitri M. Vasilkov ${ }^{1}$ \\ Belarus State University, Faculty of Applied Mathematics and Informatics, Prospekt F. Skarina 4, \\ 220050 Minsk, Belarus \\ e-mail: on.kovalev@zib-berlin.de
}

Abstract: Using the partial order technique, we describe a subclass of objective functions taking their optimum at the greedy point of a given feasible polyhedron in $\mathbf{R}^{n}$.

Key Words: Canonical order, greedy algorithm, linear programming.

\section{Introduction}

The relation $\prec$ of the canonical order is defined on $\mathbf{R}^{n}$ by

$$
x<y \Leftrightarrow g_{s}(x) \leq g_{s}(y) \quad s=1 \ldots n
$$

where $g_{s}(x)=\sum_{i=1}^{s} x_{i}$. The greedy solution $x^{g}$ of the problem

$$
\max \{f(x) \mid x \in D\}
$$

is defined as the lexicographical maximum of the feasible set $D$.

As it was shown in $[1,2,3]$, optimality of the greedy solution is closely connected with existence in the feasible set a single maximum w.r.t. $\prec$. Namely, the greedy algorithm on a polymatroid always provides the optimal solution for any nonnegative linear objective function, whereas polymatroids are a unique class of polyhedra in $\mathbf{R}^{n}$ which have a single maximum w.r.t. $<$ for any ordering of variables. In [4] we've obtained conditions defining a polyhedron in $\mathbf{R}^{n}$ with

1 Supported by the Belarusian Foundatmental Rescarch Found 
a single maximum $x^{*}$ for a fixed order of variables. It was also shown that a class $\mathscr{F}$ of all continuously differentiable functions ( $C^{1}$-functions), isotone w.r.t. $\prec$, is defined by

$$
0 \leq \frac{\partial f}{\partial x_{i+1}}(x) \leq \frac{\partial f}{\partial x_{i}}(x) \quad \text { for all } \quad x \in \mathbf{R}^{n}, \quad i=1 \ldots n-1
$$

Thus, for any $f \in \mathscr{F}$ and for certain class of feasible polyhedra it holds $x^{o p t}=$ $x^{*}=x^{g}$.

Here we consider the following problem.

Problem 1: Given an arbitrary feasible polyhedron $D \in \mathbf{R}^{n}$, describe a class $\mathscr{F}(D)$ of objective functions which take their maximum at the greedy point.

Our goal is to describe a subclass in $\mathscr{F}(D)$ using the partial order technique. The main idea consists in introducing on $\mathbf{R}^{n}$ a relation $\prec^{h}$ of the generalized canonical order, and describing a family of all orders $\prec^{h}$ for which the poset $\left(D,<^{h}\right)$ has a single maximum. Since a single maximum w.r.t. $\prec^{h}$ always coincides with the greedy solution, corresponding classes of isotone functions turns to belong to $\mathscr{F}(D)$.

\section{Generalized Canonical Order}

Let $\mathscr{M}$ be a class of strictly increasing separable concave functions on $\mathbf{R}^{n}$. For $h(x)=\sum_{i=1}^{n} h_{i}\left(x_{i}\right) \in \mathscr{M}$ we denine a relation $\prec^{h}$ of the generalized canonical order by

$$
x \prec^{h} y \Leftrightarrow g_{s}^{h}(x) \leq g_{s}^{h}(y) \quad s=1 \ldots n
$$

where $g_{s}^{h}(x)=\sum_{i=1}^{s} h_{i}\left(x_{i}\right)$. Note that $\prec^{h}$ defines a partial order (the antisymmetric property follows from strict increasing of $h$ ).

We say that an order $<_{1}$ includes an order $<_{2}$ if $x<_{2} y$ yields $x<_{1} y$. It is easy to show that the lexicographical order includes any canonical order $\prec^{h}$ which, in its turn, includes the coordinate order. Moreover, the order $\prec^{h}$ approaches the lexicographical order if $h_{i}(t)=\varepsilon^{i} t$ and $\varepsilon \rightarrow 0$. Similarly, it approaches the coordinate order if $h_{i}(t)=\varepsilon^{-i} t$ and $\varepsilon \rightarrow 0$. 
Conditions defining the class $\mathscr{F}^{h} \subset C^{1}$ of isotone functions w.r.t $\prec^{h}$ can be easily obtained from (2) by substitution $x_{i}=h_{i}\left(z_{i}\right)$ :

$$
0 \leq \frac{h_{i}^{\prime}\left(x_{i}\right)}{h_{i+1}^{\prime}\left(x_{i+1}\right)} \frac{\partial f}{\partial x_{i+1}}(x) \leq \frac{\partial f}{\partial x_{i}}(x) \quad \text { for all } \quad x \in \mathbf{R}^{n}, \quad i=1 \ldots n-1 .
$$

The class $\mathscr{F}^{h}$ contains, in particlar, each function $g_{s}^{h}$ and any composition $F\left(y_{1}(x), \ldots, y_{m}(x)\right)$, nondecreasing on $y$, and with $y_{i}(x) \in \mathscr{F}^{h}$.

Our aim is to describe a family of all orders $\prec^{h}$ such that $\mathscr{F}^{h} \subset \mathscr{F}(D)$. Note that if $\left(D, \prec^{h}\right)$ has a single maximum $x^{*}$ then $x^{*}=x^{o p t}$ for any $f \in \mathscr{F}^{h}$. On the other hand, if $x^{*}$ exists, then $x^{*}=x^{g}$, since the lexicographical order includes $\prec^{h}$. Thus, the problem is reduced to the next one.

Problem 2: Given an arbitrary polyhedron in $\mathbf{R}^{n}$, describe the set $\mathscr{L}(D)$ of all $h \in \mathscr{M}$ for which $\left(D, \prec^{h}\right)$ has a single maximum.

Remark: Suppose we want to solve (1) with incomplete knowledge about the objective function $f$. All information we have is that $f$ is isotone w.r.t. some order $\left\langle^{h}\right.$. Then the functions $w_{i}(x)=h_{i}^{\prime}\left(x_{i}\right) / h_{i+1}^{\prime}\left(x_{i+1}\right)$ report how much information we have. For example, if $w_{i}(x) \equiv \varepsilon \rightarrow 0$, then condition (3) reduces to inequalities $\frac{\partial f}{\partial x_{i}}(x) \geq 0$ reporting only that $f$ is a nondecreasing function. In this sense, problem 2 may have another interpretation: how much information about the objective function is enough to solve problem (1) if the objective function is unknown?

We'll need the following lemma.

Lemma 1: The greedy solution $x^{g}$ is a single maximum of $\left(D, \prec^{h}\right)$ iff it is the optimal solution for $n$ problems

$$
\max \left\{g_{s}^{h}(x) \mid x \in D\right\} \quad s=1 \ldots n .
$$

Indeed, the optimal solution for (4) satisfies $x<^{h} x^{g}$ for all $x \in D$.

For $h \in \mathscr{M}$ define an $n$-vector $\nabla g_{s}^{h}(x)=\left(h_{1}^{\prime}\left(x_{1}\right), \ldots, h_{s}^{\prime}\left(x_{s}\right), 0, \ldots, 0\right)$ and a matrix

$$
\nabla G^{h}(x)=\left[\begin{array}{c}
\nabla g_{1}^{h}(x) \\
\vdots \\
\nabla g_{n}^{h}(x)
\end{array}\right] .
$$


Let $D=\left\{x \in \mathbf{R}^{n}: A x \leq b\right\}$ be a nondegenerate polyhedra and $A_{B}$ be a basic submatrix corresponding to $x^{g}$ ( $x^{g}$ is always a basic solution by definition).

Theorem 1: The greedy solution $x^{g}$ is a single maximum of $\left(D,<^{h}\right)$ iff

$$
\nabla G^{h}\left(x^{g}\right) A_{B}^{-1} \geq 0
$$

Proof: Problems (4) are problems with concave objective functions and linear restrictions. According to the Kuhn-Tucker conditions for problems of this kind, $x^{g}$ is the optimal solution for $\max \left\{g_{s}^{h}(x) \mid x \in D\right\}$ iff there exists a vector $\lambda \geq 0$ such that

$$
\nabla g_{s}^{h}\left(x^{g}\right)-\lambda A_{B}=0
$$

Writing (6) for every $s=1 \ldots n$, we obtain (5).

Corollary 1: The greedy solution is optimal for any $f \in \mathscr{F}^{h}$ iff the feasible polyhedron satisfies (5).

Note that condition (5) is defined by values of $h_{i}^{\prime}$ only at the greedy point. Denote $\alpha_{i}=h_{i}^{\prime}\left(x_{i}^{g}\right)$ and consider (5) as a system of inequalities w.r.t. $\alpha$ under the condition $h_{i}^{\prime}\left(x^{g}\right)>0$ :

$$
\begin{aligned}
& \sum_{i=1}^{s} \alpha_{i} \bar{a}_{i j} \geq 0 \quad s=1 \ldots n, \quad j=1 \ldots n, \\
& \alpha_{i}>0 \quad i=1 \ldots n,
\end{aligned}
$$

where $\bar{a}_{i j}$ is an element of $A_{B}^{-1}$.

Let $\mathscr{A}(D)$ be the set of feasible solutions of (7). The following theorem gives the solution of problem 2 .

Theorem 2: For an arbitrary polyhedron $D$ in $\mathbf{R}^{n}$ the set $\mathscr{L}(D)$ is always nonempty and is defined as follows

$$
\mathscr{L}(D)=\bigcup_{\alpha \in \mathscr{A}(D)}\left\{h \in \mathscr{M}: \nabla g_{n}^{h}\left(x^{g}\right)=\alpha\right\}
$$


Proof: It suffices to show that $\mathscr{L}(D)$ is nonempty, i.e. to find $h \in \mathscr{M}$ such that $\left(D,<^{h}\right)$ has a single maximum.

Let $\varepsilon>0$ satisfies the following: if $x$ and $y$ are basic solutions and $x \neq y$, then

$$
\varepsilon<\left|x_{i}-y_{i}\right|<\frac{1}{\varepsilon}
$$

Define $h(x):=\alpha x$ with $\alpha:=\left(1, \frac{\varepsilon^{2}}{2},\left(\frac{\varepsilon^{2}}{2}\right)^{2}, \ldots,\left(\frac{\varepsilon^{2}}{2}\right)^{n-1}\right)$. Then $x^{g}$ is a single maximum w.r.t. $\prec^{h}$ by lemma 1 . Indeed, let $x$ be any basic solution and let $i=\min \left\{1 \leq j \leq n: x_{j} \neq x_{j}^{g}\right\}$. Then for any linear $f(x)=c x \in \mathscr{F}^{h}$ (including $\left.g_{s}^{h}(x)\right)$ we have

$$
\begin{aligned}
c\left(x^{g}-x\right) & =c_{i}\left(x_{i}^{g}-x_{i}\right)+c_{i+1}\left(x_{i+1}^{g}-x_{i+1}\right)+\cdots+c_{n}\left(x_{n}^{g}-x_{n}\right) \\
& \geq c_{i} \varepsilon-c_{i+1} \frac{1}{\varepsilon}-\cdots-c_{n} \frac{1}{\varepsilon} \\
& \geq c_{i}\left(\varepsilon-\frac{\varepsilon^{2}}{2} \frac{1}{\varepsilon}-\left(\frac{\varepsilon^{2}}{2}\right)^{2} \frac{1}{\varepsilon}-\cdots-\left(\frac{\varepsilon^{2}}{2}\right)^{n-1} \frac{1}{\varepsilon}\right)>0 .
\end{aligned}
$$

\section{Some Examples}

Consider a problem

$$
\max \{f(x): a x \leq b, 0 \leq x \leq d\}
$$

where $a, b>0$. It is easy to see that the greedy solution for (8) is of the form

$$
x^{g}=\left(d_{1}, \ldots, d_{k-1}, \frac{1}{a_{k}}\left(b-\sum_{i=1}^{k-1} a_{i} d_{i}\right), 0, \ldots, 0\right) .
$$

where $1 \leq k \leq n$. The problem is to describe $\mathscr{L}(D)$, i.e. to find all $h \in \mathscr{M}$ such that $x^{g}$ is optimal for any $f \in \mathscr{F}^{h}$. 
Corollary 2: A function $h \in \mathscr{M}$ belongs to $\mathscr{L}(D)$ iff for any $i$ and $j$ such that $1 \leq i<k<j \leq n$, it holds

$$
\frac{h_{i}^{\prime}\left(x_{i}^{g}\right)}{a_{i}} \geq \frac{h_{k}^{\prime}\left(x_{k}^{g}\right)}{a_{k}} \geq \frac{h_{j}^{\prime}\left(x_{j}^{g}\right)}{a_{j}} .
$$

Proof: The basic matrix and its inverse corresponding to $x^{g}$ are the following

$$
\begin{aligned}
& A_{B}=\left[\begin{array}{ccccc} 
& I & & 0 & \\
& & & & \\
a_{1} & \cdots & a_{k} & \ldots & a_{n} \\
& & & &
\end{array}\right] \quad \text { and } \\
& A_{B}^{-1}=\left[\begin{array}{ccccc} 
& I & & 0 \\
-\frac{a_{1}}{a_{k}} & \cdots & \frac{1}{a_{k}} & \ldots & \frac{a_{n}}{a_{k}} \\
& 0 & & -I
\end{array}\right] .
\end{aligned}
$$

Hence, as theorem 2 implies, $h \in \mathscr{M}$ belongs to $\mathscr{L}(D)$ iff

$$
\begin{array}{ll}
h_{i}^{\prime}\left(x_{i}^{g}\right)-\frac{a_{i}}{a_{k}} h_{k}^{\prime}\left(x_{k}^{g}\right) \geq 0 \quad \text { for } i<k \quad \text { and } \\
-h_{j}^{\prime}\left(x_{i}^{g}\right)+\frac{a_{j}}{a_{k}} h_{k}^{\prime}\left(x_{k}^{g}\right) \geq 0 \quad \text { for } k<j .
\end{array}
$$

Acknowledgement: We are very grateful to the unknown referees for useful comments and remarks. We would espeically like to thank the referee who proposed a short and elegant proof of theorem 2 .

\section{References}

[1] Edmonds J (1970) Submodular functions, matroids and certain polyhedra. In: Combinatorial Structures and their Applications. Gordon and Breach, New York 69-87 
[2] Zimmermann U (1977) Some partial orders related to boolean optimization and the greedy algorithm. Annals of Discrete Mathematics 1:539-550

[3] Kovalev M (1987) Matroids in discrete optimization. Belarusian University Press, Minsk (in Russian)

[4] Kovalev MM, Vasilkov DM The canonical order and greedy algorithms. European Journal of Operational Research 